\title{
The effect of vitamin D on MCF-7 breast cancer cell metabolism
}

\author{
Saracligil B, Ozturk B, Unlu A, Abusoglu S, Tekin G \\ Department of Biochemistry, Faculty of Medicine, Selcuk University, Konya, Turkey. beyzaozel@yahoo.com
}

\begin{abstract}
The role of vitamin $D$ in calcium absorption and bone health is known. The studies revealed that vitamin D modulates breast cancer cell growth and it is also associated with a reduced breast cancer risk. The primary objective of this study was to highlight the metabolic effect of Vitamin D on MCF-7 breast cancer cell line. For that purpose, we checked the apoptosis, energy, amino-acid and acylcarnitine levels in cancer cells, that the study propose, that $1 \alpha, 25(\mathrm{OH})_{2} \mathrm{D}_{3}$ could inhibit cell growth in a dose and time dependent manner. $\mathrm{IC}_{50}$ dose was calculated as $145 \mathrm{nM}$ for vitamin D. We observed the apoptosis level in vitamin D groups, which were 18, 28 and $38.5 \%$ at 24,48 and 72 hours, respectively. During metabolic screening analysis, it was observed that glutamine, methionine and glutamic acid levels were treated more by Vitamin D groups in cell line and also, that acylcarnitine level was increased in 24 and 48 hour groups when compared to the control, but decreased in 72 hours. Further studies are needed to analyze the role of amino acids and acylcarnitines for early apoptosis and cancer metabolism (Tab. 2, Fig. 4, Ref. 24). Text in PDF www.elis.sk. KEY WORDS: vitamin D, apoptosis, energy, amino-acid and acylcarnitine levels.
\end{abstract}

\section{Introduction}

Breast cancer is the most frequently diagnosed cancer worldwide and the leading cause of cancer death among females, with an estimated 1.7 million cases and 521,900 deaths in 2012(1). The first suggestion that vitamin D could influence cancer came in 1980 when researchers (2) proposed that the high rate of colon cancer seen in the Northern U.S. compared to the Southern U.S. was due to the UV light-induced production of vitamin D in the skin. A growing body of literature emerging from epidemiologic and laboratory studies led to the hypothesis that vitamin D could reduce breast cancer risk.

Vitamin D is well known as a modulator of calcium and bone metabolism. For the past three decades, an abundant evidence has accumulated to indicate that the active form of vitamin $\mathrm{D}, 1 \alpha, 25$ dihydroxyvitamin $\mathrm{D}_{3}, 1 \alpha, 25(\mathrm{OH})_{2} \mathrm{D}_{3}$, or calcitriol, possesses many actions not associated with calcium and bone metabolism. They include anti-proliferation, anti-angiogenesis, pro-apoptosis, prodifferentiation, and immune regulation in a cell and tissue-specific manner (3-6).

Tumor regression occurs when the rate of cell death exceeds that of cell proliferation. Cell death can be regulated by different mechanisms such as necrosis or apoptosis. Apoptosis is a highly regulated natural process that can remove undesirable, redundant, or damaged cells from multicellular organisms in an orderly, noninflammatory way. Agents that promote apoptosis in tumors are currently considered as powerful tool for cancer therapeutics. (7).

Department of Biochemistry, Faculty of Medicine, Selcuk University, Konya, Turkey

Address for correspondence: $\mathrm{B}$. Saracligil, $\mathrm{PhD}$, Department of Biochemistry, Faculty of Medicine, Selcuk University, Konya, Turkey.
The number of diseases that could be detected by the multiplex analysis of acylcarnitine and amino acid increased at a greater extent by the introduction of MS/MS.

Cancer cells have several specific metabolic features, which have been explored for targeted therapies. One of the first described was the Warburg effect (8) a shift from oxidative phosphorylation in mitochondria towards glycolysis. Mass spectrometry based analysis (NBS) is used for metabolic screening in blood samples impregnated on the papers $(9,10)$. This method provides us with the information about cell metabolism when adapted to cell culture (11).

Adenosine triphosphate (ATP) molecules provide energy for all cellular functions. Energy consumption from metabolic activities in normal cells relies primarily on mitochondrial oxidative phosphorylation, which is efficient and generates more ATP than glycolysis. However, one of the metabolic features of cancer cells is to avidly take up glucose for aerobic glycolysis. Therefore, understanding the features and complexity of the cancer energy metabolism will help to develop new approaches in early diagnosis and effectively target cancer therapy. The aim of this study was to investigate the metabolic profiling of these cells.

\section{Materials and methods}

All cells were cultured at $37^{\circ} \mathrm{C}$ and $5 \% \mathrm{CO}_{2}$ in humidified atmosphere. Human breast cancer cell lines, MCF-7, were obtained from American Type Culture Collection (ATCC, US) and were grown in DMEM (Dulbecco's Modified Eagle Medium) supplemented with $10 \%$ fetal bovine serum (FBS), $100 \mathrm{IU} / \mathrm{mL}$ penicillin, and $100 \mathrm{mg} / \mathrm{mL}$ streptomycin.

All supplements were purchased from Sigma including $1 \alpha$, $25(\mathrm{OH})_{2} \mathrm{D}_{3}$ We used xCELLigence System to monitor the cell 
101-106

Tab. 1.The distribution of different phases of MCF-7 cell cycle after $1 \alpha, 25(\mathrm{OH})_{2} \mathrm{D}_{3}$ treatment determined by flow cytometry with Annexin $\mathrm{V}$ FITC and 7-AAD staining.

\begin{tabular}{lccc}
\hline & $\begin{array}{c}\text { 7-IADD Negative, } \\
\text { Annexin V Positive (\%) }\end{array}$ & $\begin{array}{c}\text { 7-ADD Negative, } \\
\text { Annexin V Positive (\%) }\end{array}$ & 7-ADD Positive, \\
\hline Control 24h & 87.8 & 1.1 & 18 \\
\hline Vitamin D 24h & 74.1 & 1.1 & 0.5 \\
\hline Control 48h & 90.6 & 28.6 & 0.8 \\
\hline Vitamin D 48h & 67.4 & 0.8 & 2.8 \\
\hline Control 72h & 82.4 & 38.5 \\
\hline Vitamin D 72h & 52.3 & 0.9 \\
\hline
\end{tabular}

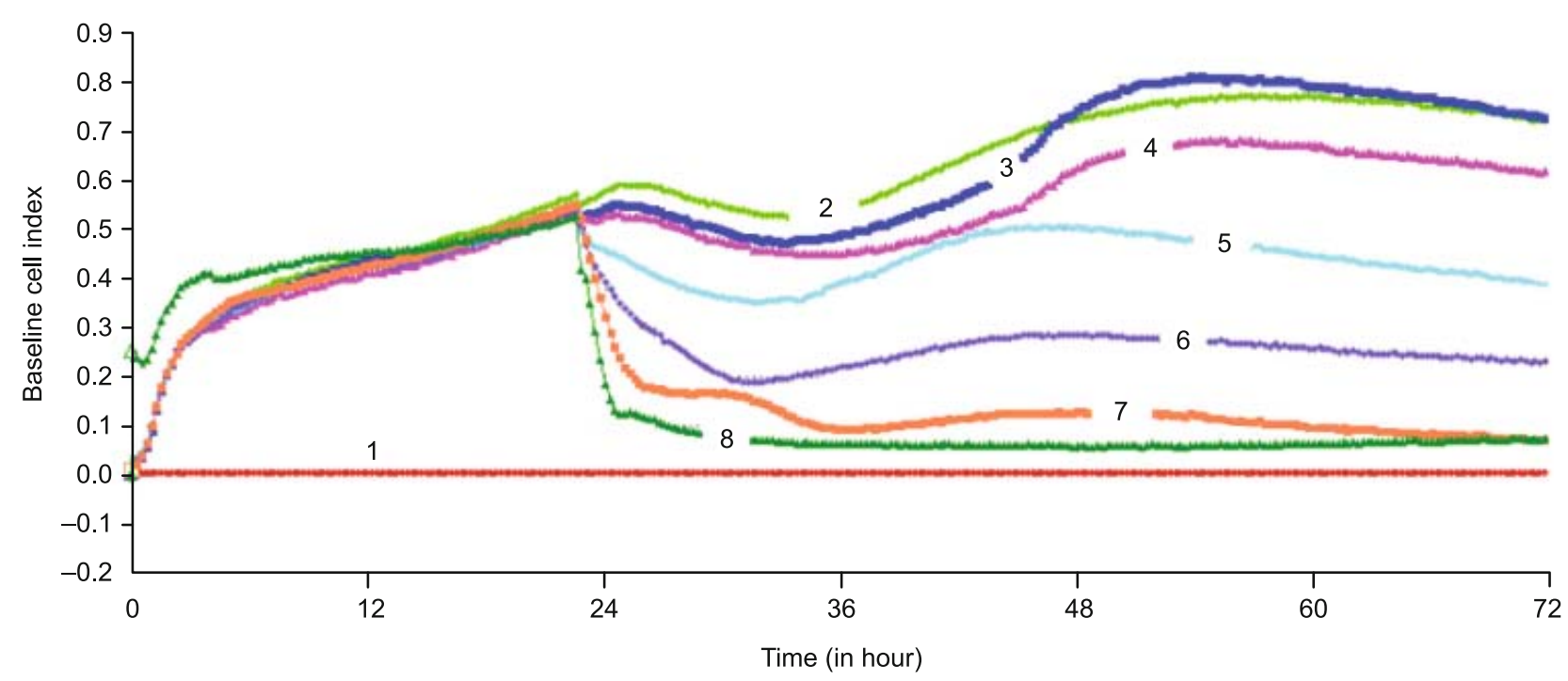

Fig. 1. The dose dependent inhibitory effects of vitamin D on the growth of MCF-7 cells. 1 - DMEM, 2 - CONTROL, 3 - ETHANOL, 4 - $10 \mathrm{nM}$ vitamin $D, 5-100 \mathrm{nM}$ vitamin $\mathrm{D}, 250 \mathrm{nM}$ vitamin $\mathrm{D}, 500 \mathrm{nM}$ vitamin $\mathrm{D}, 1000 \mathrm{nM}$ vitamin $\mathrm{D}$.

viability. The xCELLigence System (Roche Diagnostics GmbH, Penzbeerg, Germany) monitors cellular events in real time without the incorporation of labels.

The system measures electrical impedance across interdigitated micro-electrodes integrated on the bottom of tissue culture E-plates. Changes in cell number were observed (followed) every 15 min up to 72 hours at $10,25,50,125,250,500,1000 \mathrm{nM}$ concentrations of Vitamin D. Cell Protein Analysis was performed according to Lowry Method by Spectrometer( Perkin Elmer Lambda $25 \mathrm{UV} / \mathrm{Vis}, \mathrm{US})$.

\section{Cell energy level}

The energy levels of the cells were measured as reported by Cimen with some modification.(12). The analyses were run by HPLC (Shimadzu DGU-20A3R,US) Sample volumes $50 \mu \mathrm{M}$ were separated using a $5 \mu \mathrm{m}$ GES C18 column (Vertical VertiSep ${ }^{\mathrm{TM}}$, 4.6 x 150 $\mathrm{mm}$ ). The mobile phase of $160 \mathrm{mMKH}_{2} \mathrm{PO}_{4}$ and $100 \mathrm{mMKCI}, \mathrm{pH} 6.5$. All measurements were based on absorption of light at $254 \mathrm{~nm}$ run.

\section{Apoptosis analysis}

MCF-7 cell apoptosis was analysed using a flow cytometer with Annexin V-FITC (fluorescein isothiocyanate) and 7AAD (7-Aminoactinomycin D) staining kit (BD Biosciences) to distin- guish early apoptotic from necrotic cells as previously described (13). Briefly, one day and two days after the $145 \mu \mathrm{M} 1 \alpha, 25(\mathrm{OH})_{2} \mathrm{D}_{3}$ treatment, MCF-7 cell apoptosis was analyzed using a flow cytometer with Annexin V-FITC and 7AAD staining.

\section{Aminoacid and acylcarnitine levels}

The NBS assay kit from Chromsystems (Germany), originally developed for quantification of amino acids and acylcarnitines in dried blood samples, (Rashed et al, 1997), was adapted for use with cultured cells. For this assay, every dose and control group cells $\left(10^{6}\right)$ cultured at $100 \mathrm{~cm}^{2}$ petri dishes. Cells were harvested and trypsinized at 24,48 , and 72 hours after treatment. Cells were washed twice with PBS and cell lyses buffer was added. Cells were stored at $-80^{\circ} \mathrm{C}$. After the day of assay, cells were removed from $-80{ }^{\circ} \mathrm{C}$, then were disintegrated for 30 minutes by sonication, and centrifuged for $30 \mathrm{~min}$ at $14,000 \mathrm{rpm}$. The supernatant was collected after passing through the filter (after filtration) paper saturated with NBS and was left to stand overnight. Further, samples were analyzed by LC/MS-MS (API 3200, US).

\section{Statistical analysis}

The data were analyzed by non-parametric Mann-Whitney U test (SPSS 18 Software). 
Tab. 2. Effect of vitamin D on MCF-7 cells energy level.

\begin{tabular}{|c|c|c|}
\hline Time & Control Group ATP $\mu$ mol/g Median (min-max) & Vitamin D Group ATP $\mu$ mol/g Median (min-max) \\
\hline $24 \mathrm{hr}$ & $3.27(2.64-3.91)$ & $1.23(1.15-1.31)^{*}$ \\
\hline $48 \mathrm{hr}$ & $1.46(1.15-1.69)$ & $1.00(0.86-1.14)^{*}$ \\
\hline \multirow[t]{2}{*}{$72 \mathrm{hr}$} & $1.00(1.00-1.06)$ & $1.03(1.00-1.13)$ \\
\hline & Control Group ADP $\mu$ mol/g Median (min-max) & Vitamin D Group ADP $\mu \mathrm{mol} / \mathrm{g}$ Median $(\min -\max )$ \\
\hline $24 \mathrm{hr}$ & $5.09(3.82-5.73)$ & $3.11(2.77-3.23)^{*}$ \\
\hline $48 \mathrm{hr}$ & $5.00(4.62-5.77)$ & $3.28(3.07-3.71)^{*}$ \\
\hline \multirow[t]{2}{*}{$72 \mathrm{hr}$} & $5.56(5.35-5.88)$ & $6.10(5.53-6.20)$ \\
\hline & Control Group AMP $\mu$ mol/g Median (min-max) & Vitamin D Group AMP $\mu$ mol/g Median (min-max) \\
\hline $24 \mathrm{hr}$ & $9.13(8.73-9.64)$ & $7.88(6.69-8.00)^{*}$ \\
\hline $48 \mathrm{hr}$ & $10.57(9.62-11.46)$ & $13.86(13.71-14.36)^{*}$ \\
\hline $72 \mathrm{hr}$ & $46.59(45.18-48.94)$ & $58.06(57.07-58.93)^{*}$ \\
\hline
\end{tabular}

$* \mathrm{p}<0.05$

A
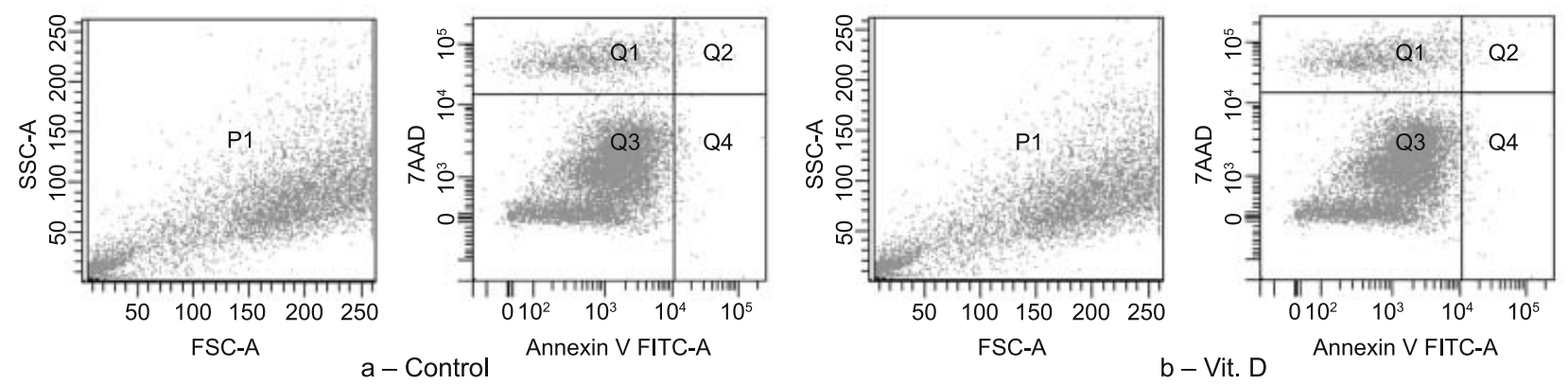

(B)
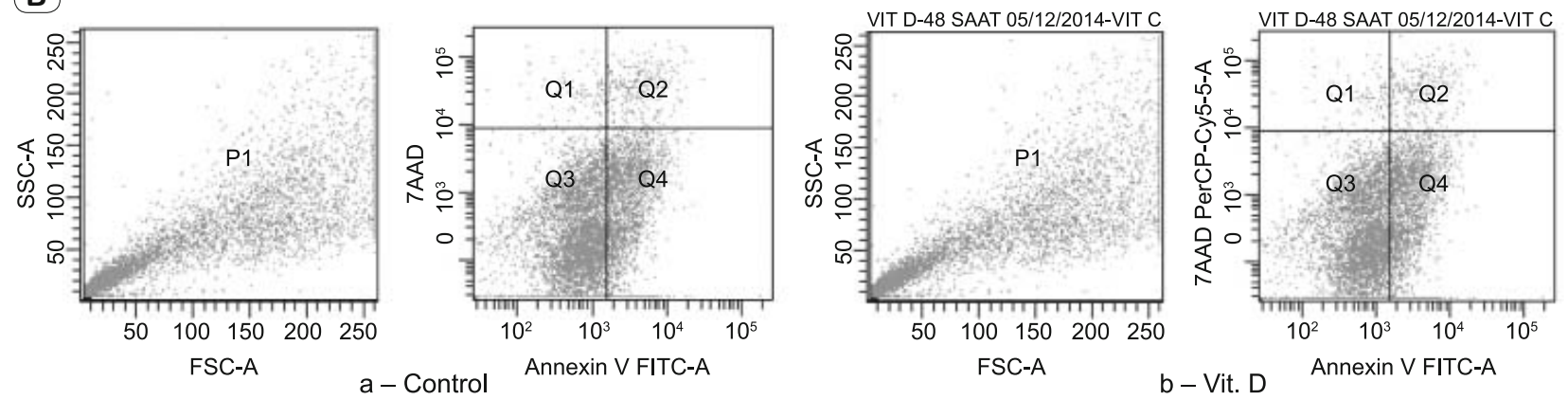

(C)
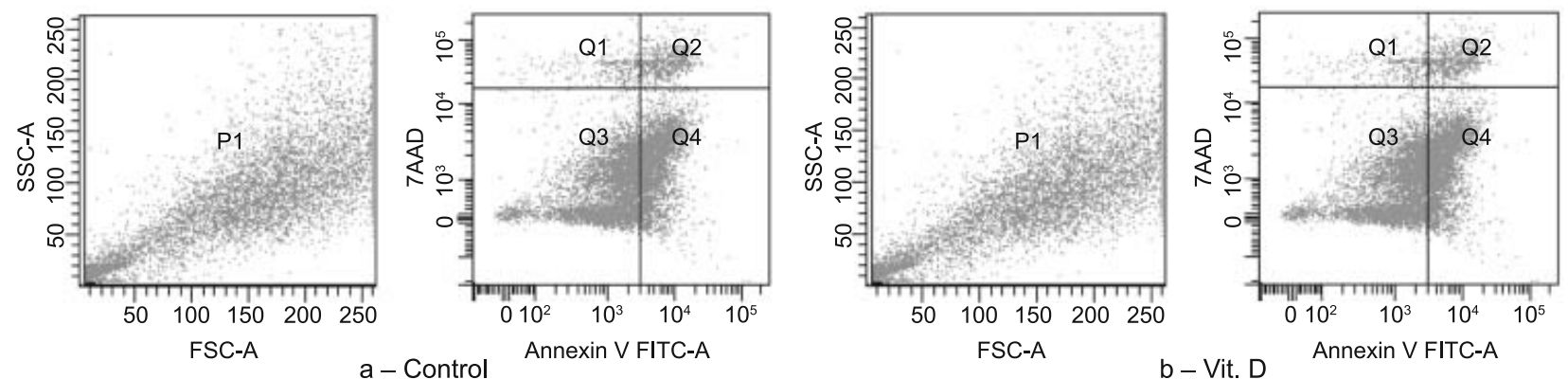

Fig. 2. Effects of $1 \alpha 25(\mathrm{OH})_{2} \mathrm{D}_{3}$ on MCF-7 cell apoptosis analyzed by flow cytometry with Annexin V-FITC, 7-AAD staining, Annexin V-FITC in conjunction with 7-AAD staining was used to distinguish early apoptotic (Annexin V-FITC positive, 7AAD negative; bottom right quadrant of each panel) from late apoptotic or necrotic cells (Annexin V-FITC positive, 7 AAD positive; top right quadrant of each panel). Fluorescence intensity for Annexin V-FITC is plotted on the $x$-axis, and 7 AAD is plotted on the y-axis. A - 24th, B - 48th, C - 72th hour Groups. 
101-106

\section{Results}

Effect of vitamin D on MCF-7 cells proliferation

The effect of vitamin D on MCF-7 growth was analyzed in the relation to dose and time. As presented in Figure 1, vitamin $\mathrm{D}$ caused a dose dependent inhibition of cell growth. It has been also observed that Vitamin D at 500 and $1000 \mathrm{nM}$ doses showed direct toxic effect on MCF-7 cells. IC50 was calculated as 145 $\mathrm{nM}\left(\mathrm{r}^{2}=0.99\right)$ with reference to the proliferation curve of the Vitamin D applied cell.

\section{Cell energy level}

ATP, Adenosine diphosphate (ADP) and Adenosine monophosphate (AMP) results of MCF-7 cells, to which Vitamin D was applied, were obtained at 24, 48 and 72 hours through HPLC device and they were verified using cell protein levels. The analysis was repeated six times.

\section{Effects of $1 \alpha, 25(\mathrm{OH})_{2} D_{3}$ on $\mathrm{MCF}-7$ cell apoptosis}

To compare the apoptotic response induced by $1 \alpha, 25(\mathrm{OH})$ in MCF-7 cells, flow cytometry analysis coupled with staining cells with Annexin V (Annexin V-FITC) and 7-ADD was utilized. The quantitative numerical distribution of apoptotic cells from this analysis is presented in the Table 1. When Vitamin D in our groups was compared to the control groups, it was observed that the apoptosis level, which was $18 \%$ at 24 hour (Annexin V Positive, 7-AAD Negative) was respectively $28 \%$ at 48 hour (Annexin V Pozitif,7AAD Negative), and $38.5 \%$ at 72 hour.

\section{Amino acid and Acyl carnitine Level}

During this analysis, we compared the amino acid and acyl carnitine levels of Vitamin D and the control cell groups by using NBS analysis at LC MS/MS device. We observed that in 24 and 48 hour treated group, glutamine, methionine and glutamic acid levels were used more in the Vitamin D groups than the one in the control groups. This situation took a reverse path in the 72th group. However, the total aclycarnitine level decreased at the 72th hour while it increased at the 24th and 48th hours when compared to the control group.

\section{Discussion}

The focus of this study was to investigate the metabolic activities in addition to anti-proliferative and pro-apoptotic effect of Vitamin $\mathrm{D}_{3}$ in the MCF-7 breast cancer cell. Linked to dose and time, we observed the anti-proliferative effects of vitamin $\mathrm{D}$ with real time cell detecting system (Fig. 1).

A reduction in growth rate of melanoma culture cells that were first treated by Colston (1981) with $1,25(\mathrm{OH})_{2} \mathrm{D}$ was seen dependent to dose (14). The growth inhibitory effect of $1,25(\mathrm{OH})_{2}$ $\mathrm{D}$ on cancer issues including, but not limited to colon, breast and prostate is also clear (15-17). We observed that Vitamin D was inhibiting cell growth in our study when compared to the control group, dependent on the dose and time (Fig. 2). IC50 dose, which was calculated using the aforementioned values was found as 145 $\mathrm{nM}$ similar to the literature (6).
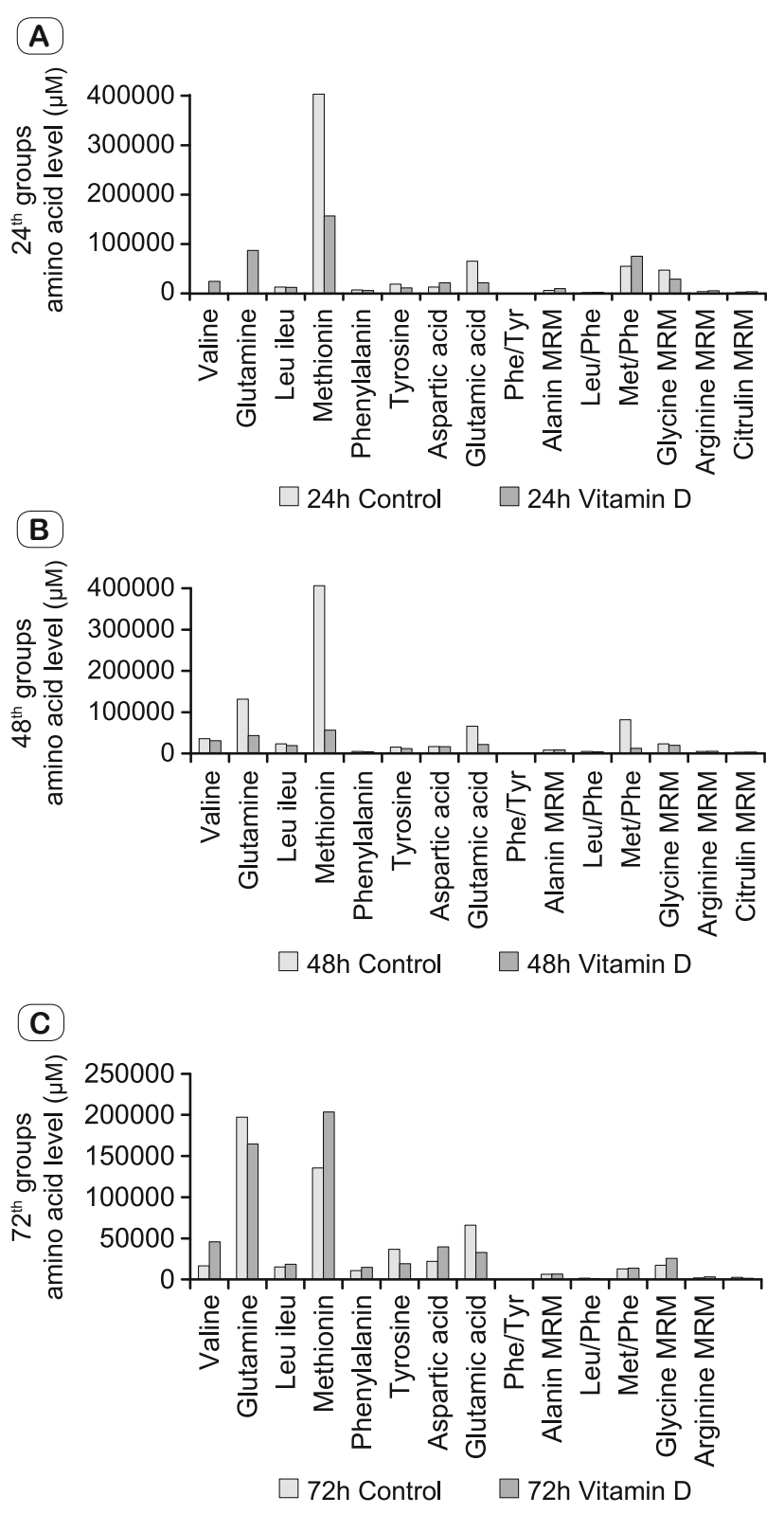

Fig. 3. Overview of Amino-acid Level as dedected by LC-MS/MS on MCF-7 cell Lines undergo inVitamin D treatment. A - 24th Groups, B - 48th Groups, C - 72th Groups.

Apoptosis is a well-designed natural process, which removes undesirable or damaged cells in multi cell organisms in non-inflammatory way. The cancer therapy becoming more efficient with the in vivo or in vitro when the use of radiotherapy or anticancer drugs is related to rising apoptosis markers (7). MCF-7 cells expresses the genes, which play a critical role for Vitamin D signal pathway and metabolism and 1,25(OH)2D3 application inhibited cell growth and proliferation and thereby increased apoptosis (18). Our results also confirmed the existing literature. Apoptosis was in an increasing trend: it was $18 \%$ at the 24 th hour (Annexin V positive, 7-AAD negative) respectively $28 \%$ at the 48 th hour (Annexin V positive, 7-AAD negative), and $38.5 \%$ at the 72 th hour (Tab. 1). 


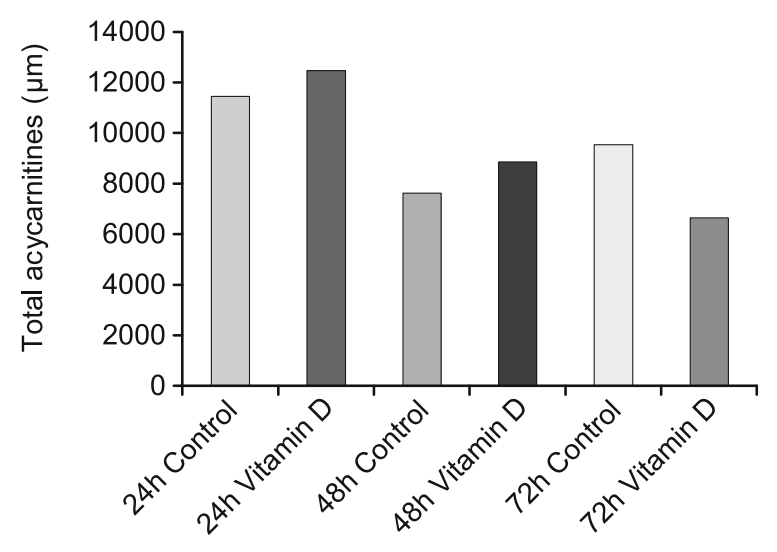

Fig. 4. Total Acylcarnitines Level of Vitamin D Groups.

ATP is an energy transporter for energy requiring reactions occurring in cell. AMP levels in cytosol represent a better indicator than ATP concentration for ATP usage rate.

AMP concentration is defined with the equilibrium point of adenylate kinase reaction.

At the equilibrium point during all energy requiring reactions like hydrolysis of ATP into ADP, both ADP and AMP contents increase in cytosol. However, ATP is found in higher quantity in comparison to AMP or ADP, for this reason any small reduction in ATP concentration results in a higher increase in AMP pool (Smith, Marks, Lieberman, Marks, \& Marks, 2005). AMP functions are to be an energy sensor and a metabolism regulator.

When ATP production is unable to meet the requirements, a larger part of a cell's adenine nucleotide pool is in shape of AMP. AMP thereafter stimulates ATP producing metabolic pathways. When 24th, 48th and 72nd hour groups from Vitamin D applied cells were compared to control groups, we observed that the AMP level were increasing by time, a clear evidence that ATP was used (Tab. 2). This lead us to think of a parallelism with increasing apoptosis rate.

Tumor cells utilize glucose, lipids or amino acids in order to grow and reproduce depending to the tumor type and evolution stage (19). To be able to grow, tumors need essential amino acids, for purine and pyrimidine synthesis amino acids like glutamine, glycine and aspartic acids and for the synthesis of membrane lipid components serine.

Therefore, they need selective amino acids. During our metabolic screening, we observed that glutamine, methionine and glutamic acid levels were used more in the Vitamin D groups than the ones in the control groups (Fig. 3). Methionine, whose crucial role in DNA synthesis and single carbon metabolism is highlighted, is an essential amino acid. Some cancer cell cultures show a growth dependent to the methionine. Methionine amount/quantity, which was seen in decrease when compared to the control groups in our study was very remarkable (Fig. 3).

Glutamine (GLN), which is largely found in blood, is making up $50 \%$ of amino acid pool GLN and whose $75 \%$ is found in skeletal muscle and most of the remaining portion in liver, is neutral and can be essential amino acid according to the circumstances (Cresci, 2005). GLN is the most important component of proteins. It contains in its structure two amine groups; purine and pyrimidine, therefore it takes part in nucleic acid synthesis as an important nitrogen conductor/carrier. Glutation (GSH/glutamyl cysteinyl-glycine), which is a side product GLN metabolism is one of the most intense antioxidants and protects normal tissue against oxidative damages. GLN, for its importance in nucleic acid synthesis is an amino acid required for the continuity of regularly dividing and reproducing cells. It has energy source and nitrogen carrier function. It is also the leading regulator of glycogenesis and protein synthesis. Last studies on cancer metabolism have offered insight to the cell reproduction and maintenance, alternative energy sources and especially the role of glutamine and other amino acids.

Proofs observed from various tests confirmed this result. It is presented that estrogen simulation was inducing glutaminolysis in breast cancer cells (20).

In our study, glutamine amount showed significant changes. In comparison to the control groups, glutamine amount decreased notably in 24th and 48th hour Vitamin D groups. The other parameter of NBS analysis carnitine profile (ACP) performs biochemical screening of fat acid irregularities and organic acid metabolism.

It is also represented that carnitines have a protective impact against toxic effects caused by cisplatin (21) and the in-cell transport is related to apoptosis (22). Besides, carnitines play a vital role in energy production and fat acid mechanism and thanks to its useful effects, nutritional variation is under study (23).

Acylcarnitines can be protective against oxidative tension by the reduction of lactic acidosis leading to ROS formation through changes at mitochondrial and cytosolic stages, or induction of antioxidant genes (24). We also observed in our study that it increased in 24th and 48th groups in comparison to the control ones but decreased in 72th (Fig. 4). The decrease in 72th group revealed that it can be linked to increasing AMP, i.e. reduced metabolic speed as well as to $38 \%$ apoptosis level.

\section{Conclusion}

It was observed that the anti-proliferative effect of Vitamin D on MCF-7 breast cancer cell was similar to literature through the real time cell electronic detection system.

Using these data, we considered that $\mathrm{IC}_{50}$ value calculated as $140 \mathrm{nM}$ would be helpful for treatment and cell culture studies. We observed that it led to reduction in energy level both at 24th group significantly and 48th group at ATP, ADP and AMP nucleotides. These data evidenced the apoptosis mechanism increasing by time and requiring energy.

The fact that Vitamin D lead to apoptosis when considered as therapeutic agent, we think that it can be used in cancer therapy mostly in breast cancer and therefore vitamin D blood level optimization is of utmost importance. Metabolic screening results were very remarkable for glutamine, glutamic acid and methionine amino acid. When it was compared to the control group, a reduction was seen in Vitamin D groups. If NBS analysis is considered to be the screening test, in our opinion, a quantitation is required 


\section{1-106}

for amino acids and acylcarnitines at the next step. In conclusion, we think that amino acids need to be analyzed in more details both as biomarker for early apoptosis and cancer nutrition and metabolism at further studies.

\section{References}

1. Torre LA et al. Global cancer statistics, 2012. CA Cancer J Clin 2015; 65 (2): 87-108.

2. Garland CF, Garland FC. Do sunlight and vitamin D reduce the likelihood of colon cancer? Int J Epidemiol 1980; 9 (3): 227-231.

3. Fleet JC et al. Vitamin D and cancer: a review of molecular mechanisms. Biochem J 2012; 441 (1): 61-76.

4. Chiang KC, Chen TC. Vitamin D for the prevention and treatment of pancreatic cancer. World J Gastroenterol 2009; 15 (27): 3349-3354.

5. Ylikomi T et al. Antiproliferative action of vitamin D. Vit Horm 2002; 64: 357-406.

6. Chiang KC, Chen TC. The anti-cancer actions of vitamin D. Anticancer Agents Med Chem 2013; 13 (1): 126-139.

7. Call JA, Eckhardt SG, Camidge DR. Targeted manipulation of apoptosis in cancer treatment. Lancet Oncol 2008; 9 (10): 1002-1011.

8. Koppenol WH, Bounds PL, Dang CV. Otto Warburg's contributions to current concepts of cancer metabolism. Nat Rev Cancer 2011; 11 (5): 325-337.

9. Baumgartner C, Bohm C, Baumgartner D. Modelling of classification rules on metabolic patterns including machine learning and expert knowledge. J Biomed Inform 2005; 38 (2): 89-98.

10. Chace DH, Kalas TA, Naylor EW. Use of tandem mass spectrometry for multianalyte screening of dried blood specimens from newborns. Clin Chem 2003; 49 (11): 1797-1817.

11. Halama A, Moller G, Adamski J. Metabolic signatures in apoptotic human cancer cell lines. Omics 2011; 15 (5): 325-335.

12. Cimen B et al. Effects of melatonin on 3-nitrotyrosine formation and energy charge ratio in Guinea pig kidney in LPS-induced stress. Cell Biochem Funct 2005; 23 (4): 273-277.
13. Vermes I, Haanen C, Reutelingsperger C. Flow cytometry of apoptotic cell death. J Immunol Methods 2000; 243 (1-2): 167-190.

14. Colston KW, Hansen CM. Mechanisms implicated in the growth regulatory effects of vitamin D in breast cancer. Endocr Relat Cancer 2002; 9 (1): 45-59.

15. Pilon $\mathbf{C}$ et al. 1alpha,25-Dihydroxyvitamin $\mathrm{D}(3)$ inhibits the human H295R cell proliferation by cell cycle arrest: a model for a protective role of vitamin D receptor against adrenocortical cancer. J Steroid Biochem Mol Biol 2014; 140: 26-33.

16. Chang $\mathrm{S}$ et al. miR-145 mediates the antiproliferative and gene regulatory effects of vitamin D3 by directly targeting E2F3 in gastric cancer cells. Oncotarget 2015; 6 (10): 7675-7685.

17. Zhang $\mathbf{Z}$ et al. Synergy of 1,25-dihydroxyvitamin D3 and carboplatin in growth suppression of SKOV-3 cells. Oncol Lett 2014; 8 (3): 1348-1354.

18. Ooi LL et al. Vitamin D deficiency promotes human breast cancer growth in a murine model of bone metastasis. Cancer Res 2010; 70 (5): 1835-1844.

19. Gu Y et al. Perioperative dynamics and significance of amino acid profiles in patients with cancer. J Transl Med 2015; 13: 35.

20. Forbes NS et al. Estradiol stimulates the biosynthetic pathways of breast cancer cells: detection by metabolic flux analysis. Metab Eng 2006; 8 (6): 639-652.

21. Altun ZS et al. Protective effects of acetyl-L-carnitine on cisplatin cytotoxicity and oxidative stress in neuroblastoma. Neurochem Res 2010; 35 (3): 437-443.

22. Mazzarelli $\mathbf{P}$ et al. Carnitine palmitoyltransferase I in human carcinomas: a novel role in histone deacetylation? Cancer Biol Ther 2007; 6 (10): 1606-1613.

23. Flanagan JL et al. Role of carnitine in disease. Nutr Metab (Lond) 2010; 7: 30 .

24. Jones LL, McDonald DA, Borum PR. Acylcarnitines: role in brain. Prog Lipid Res 2010; 49 (1): 61-75.

Received September 26, 2016. Accepted November 3, 2016. 